\title{
Antioxidative effect of Capsicum oleoresins compared with pure capsaicin
}

\author{
Maksimova Viktorija ${ }^{1}$, Koleva G. Liljana ${ }^{2}$, Ruskovska Tatjana ${ }^{1}$, Cvetanovska \\ Ana $^{3}$, Gulaboski Rubin ${ }^{1}$ \\ ${ }^{I}$ Faculty of Medical Sciences, "Goce Delčev" University, Krste Misirkov bb, POB 201, 2000 Štip, Republic of \\ Macedonia, \\ ${ }^{2}$ Agricultural faculty, "Goce Delčev” University, Krste Misirkov bb, POB 201, 2000 Štip, Republic of \\ Macedonia \\ ${ }^{3}$ Institute of Biology, Faculty of Natural Sciences and Mathematics, "Ss. Cyril and Methodius" University, \\ Arhimedova 5, Skopje, Republic of Macedonia
}

\begin{abstract}
Capsicum annum L, or hot peppers are unique among the plant species, because of their ability to synthesize capsaicin. Capsaicin is an alkaloid which has been known for its analgesic, antireumatic, antiseptic, antidiabetic and few more pharmacological properties. Its antioxidative potential is also a subject of many experiments, in the last few years. The aim of this study is to examine the antioxidant potential of capsaicin and capsicum oleoresins produced from Capsicum sp. cultivated in R. of Macedonia.

This experiment comprises four different genotypes of Capsicum annuum L., which were used for obtaining ethanolic oleoresins. Their antioxidant potential was measured and compared to the antioxidative potential of the pure capsaicin standards. As a method for measuring the total antioxidant capacity was used FRAP (Ferric reducing antioxidant potential) method. This is a simple photometric method for estimation of in vitro antioxidative potential which is expressed as $\mu \mathrm{mol} / \mathrm{L} \mathrm{Fe} e^{2+}$.

The results from this study are showing a very good correlation between antioxidant potential of capsaicin and capsicum oleoresins.

This confirms that antioxidative potential of hot peppers does not come only from the vitamins and phenolic compounds in them, but capsaicinoids are also included.
\end{abstract}

KEY WORDS: antioxidants, capsaicin, FRAP, fruit, hot peppers.

\section{INTRODUCTION}

Pepper fruits (Capsicum annuum L.) are important vegetables used as vegetable foods, spices or dry fruits intended for isolation of capsaicin [1,2]. Peppers are a good source of vitamins $C$ and E [3, 4] as well as some of the carotenoids (provitamin A) as compounds with well-known antioxidant properties [5-7]. Capsaicinoids refers to a group of pungent compounds found in chilli peppers. Hot cultivars are rich in capsaicinoids, alkaloids with pharmacological properties giving the specific taste to pepper fruit $[4,8]$. Interest in their biological activity is increasing. Previous studies have indicated that red pepper and capsaicinoid decrease blood cholesterol concentration, [9-11] possibly mediated by inhibition of intestinal cholesterol absorption [12]. Capsaicinoids have also been shown to be effective in weight reduction mediated by enhancing $\beta$-oxidation of fatty acids in vivo and increasing adrenergic activity and energy expenditure [13]. Accumulated evidence has also demonstrated that capsaicinoid has potential beneficial effect on the human cardiovascular system $[14,15,16]$. It has also been reported that capsaicinoid possesses antitumor activity [17, 18]. Capsaicinoids have been shown to possess antioxidant activity with an ability to prevent excessive formation of ROS (reactive oxygen species) [19, 20]

There are few reports on the antioxidant activity of capsaicin [21, 22]. The antioxidant activities of this compound may be the result of the presence of the groups in the phenolic ring (a methoxy group in ortho position to $\mathrm{OH}$ ) of capsaicinoids and ferulic acid ester, which influenced the antioxidant properties. This is in agreement with Henderson et al. [21], who showed that the amide group present in capsaicin does not play a major role in its antioxidant activity under free radical oxidation conditions, that the antioxidant behavior for capsaicin was due primarily to the phenolic moiety in the molecule, and that the main product of capsaicin oxidation is its dimersdicapsaicin. A comparison of the results of antioxidant activities of pure capsaicin and Capsicum extracts obtained in the present studies showed that the antioxidant activity of oleoresins is dependent of the concentration of capsaicin. 
The aim of the present work was to complete the knowledge on Capsicum alkaloids. We have determined the antioxidant activity of the capsaicinoid fraction isolated from hot peppers and the antioxidant activity of the ethanolic oleoresins. The primary objective of this study was to determine which genotype has higher antioxidant potential and how it correlates with antioxidant potential of the pure capsaicin.

\section{MATERIALS AND METHODS}

2.1 Plant Material. Fruits of three hot pepper cultivars, (genotypes Vezena, Feferona, Bombona), and one mild genotype Sivrija, (as a control), were taken from the field experiment conducted in the years 2012-2013. Fruits were harvested at the stage of full ripeness (red) [22, 23]. After the fruits have been washed and the seeds removed, fresh pepper fruits were cut and dried at a room temperature, in a dark and dry place for about two weeks. They were dried to constant weight, and the percent of water in them was counted. Dried fruits of the peppers were grounded and kept in an exsiccator.

2.2 Method of extraction. For the extraction of oleoresin we have used the maceration method [24]. Process of extraction with vacuum filtration was made using $0,2 \mathrm{~g}$ of grounded peppers in $25 \mathrm{ml}$ of extraction solvent. According to the literature a few organic polar and non polar solvents can be used for capsaicin extraction. We have tried to use acetone and ethanol, but acetone has shown as an inappropriate solvent for spectrophotometric measurements on wavelength of $280 \mathrm{~nm}$. Therefore, in the focus of this experiment were taken only the ethanolic extracts. Maceration process was performed in volumetric flasks for 5 hours, on temperature of $50^{\circ} \mathrm{C}$. Separation of extracts from the powder was conducted with vacuum filtration using a Buchner funnel and a water vacuum pump. Final extracts for this experiment were diluted 2:25 with the same solvent.

2.3 Method for quantification of capsaicin. Concentrations of capsaicin in the standard solutions and in ethanolic extracts were measured by one of the cheapest method for quantification of capsaicin, the UV/VIS spectrometric method $[25,26]$. Measurements of the concentration of capsaicin in the extracts were performed through their absorbencies measured on UV/VIS spectrometer model Cary 100, 9.0 wavelength of $280 \mathrm{~nm}$.

2.4 Method for measuring the antioxidant potential of capsaicin. As a method for measuring the total antioxidant capacity (TAC) of the pure capsaicin or Capsicum extracts was used the in vitro spectroscopic method, FRAP (Ferric Reducing Antioxidant Power) assay [27]. This method is based on the reduction of the $\mathrm{Fe}^{3+}$ ions to $\mathrm{Fe}^{2+}$ ions, when they are captured in TPTZ (2,4,6-Tri(2-pyridinyl)-1,3,5-triazine), under the influence of the antioxidant (reductance) in the system. The reaction in this assay is running under acidic conditions. $\mathrm{FeSO}_{4}$ has been used as a standard solution in this measuring, so the results are expressed as $\mu \mathrm{mol} / \mathrm{L} \mathrm{Fe}^{2+}$. Detection of the final coloring of the samples on which this assay was applied was measured on wavelength of $595 \mathrm{~nm}$.

\section{RESULTS AND DISCUSSION}

The capsaicin content in the ethanolic oleoresins was determined on the basis of standard solutions of capsaicin (Sigma-Aldrich, Schnelldorf, Germany) [28]. The results for concentration of capsaicin in the samples were calculated using the linearity curve and linearity equation $y=9.484 x+0.016$, obtained from the standard solutions of capsaicin Fig. 1.

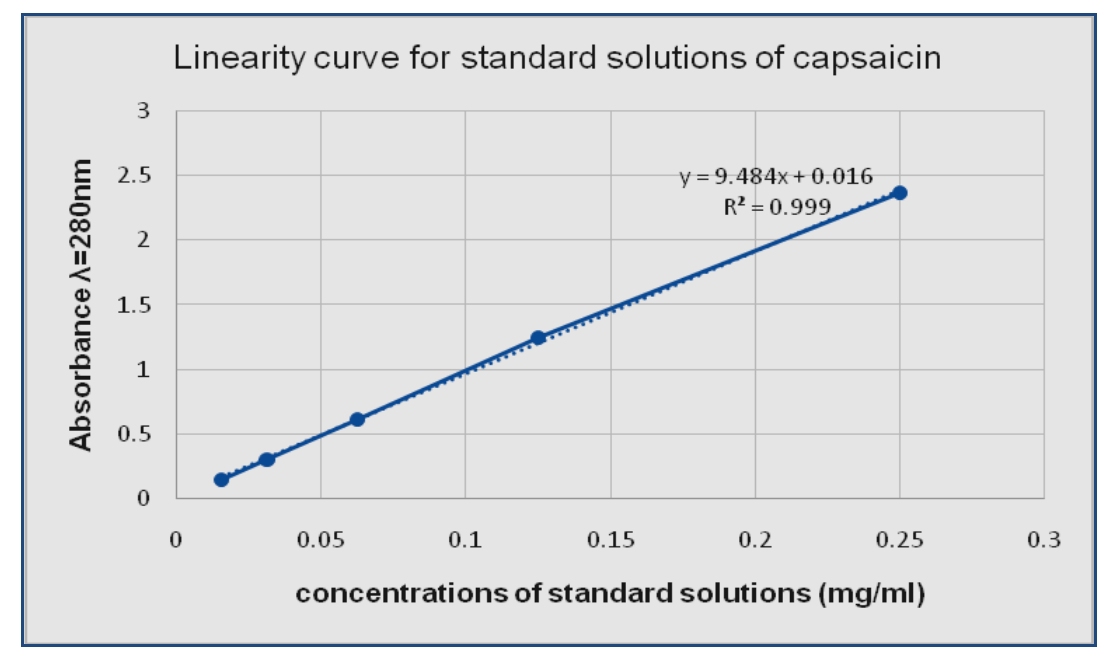

Figure1. Linearity curve for standard solutions of capsaicin

Table 1 is presenting the results of capsaicin concentration $(\lambda=280 \mathrm{~nm})$ and TAC values of the standard solutions of capsaicin, measured by spectrophotometer on wavelength of $595 \mathrm{~nm}$. 
Table 1 Capsaicin concentration and TAC of the standard solutions of capsaicin

\begin{tabular}{lccc}
\hline Standard solutions & $\begin{array}{c}\text { Concentration of } \\
\text { capsaicin }(\mathbf{m g} / \mathbf{m l})\end{array}$ & $\begin{array}{c}\text { Absorbance in FRAP } \\
\text { assay }\end{array}$ & $\begin{array}{c}\text { TAC } \\
\left(\mathbf{F e}^{\mathbf{2 +}} \boldsymbol{\mu m o l / L )}\right.\end{array}$ \\
\hline St. solution 1 & 0,25 & 1,175 & 1395 \\
St. solution 2 & 0,125 & 0,726 & 763 \\
St. solution 3 & 0,062 & 0,457 & 383 \\
St. solution 4 & 0,031 & 0,332 & 207 \\
St. solution 5 & 0,016 & 0,265 & 113 \\
\hline
\end{tabular}

The coefficients of correlation (R values) given in Fig.2 and Fig.3 are showing a very good correlation between the content of capsaicin and its total antioxidative potential. As we can see, Fig. 2 is showing the results obtained from the standard solutions of capsaicin.

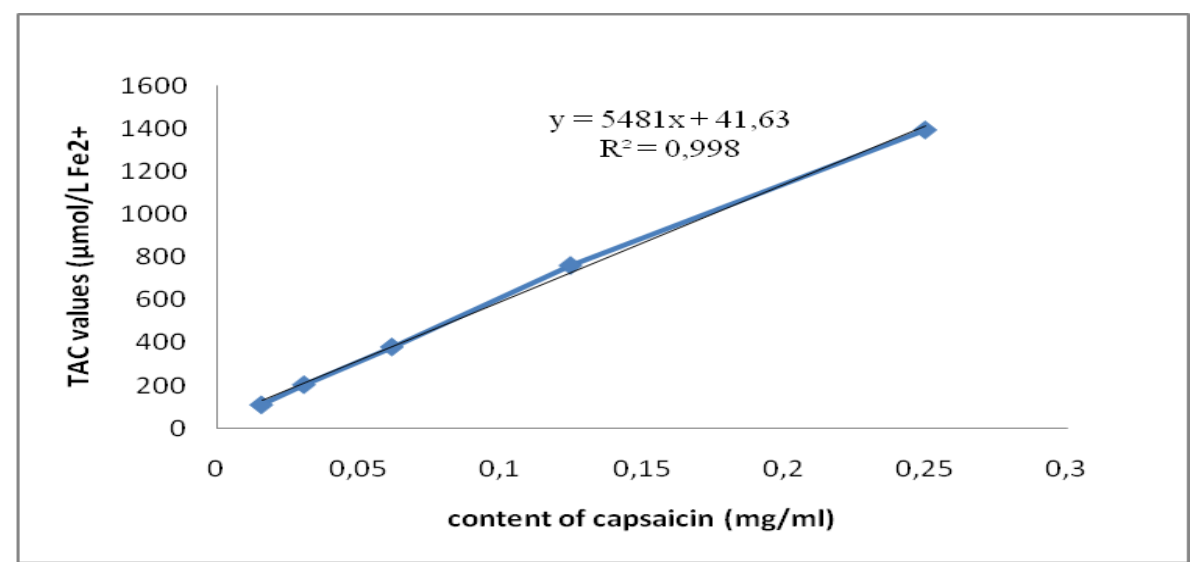

Figure 2 Correlation between capsaicin content and TACs in standard solutions of capsaicin

Table 2 is giving the results of Capsaicin concentration and TAC in the ethanolic oleoresins. Consequently on the next figure, Fig. 3 is presented the correlation between capsaicin content in oleoresins and their TAC values. But, only the hot peppers oleoresins are taken in consideration because as expected for the control there are very low antioxidant potential that cannot be measured by this method.

Table 2 Capsaicin concentration and TAC of the ethanolic oleoresins

\begin{tabular}{lccc}
\hline Extracts & $\begin{array}{c}\text { Concentration of } \\
\text { capsaicin }(\mathbf{m g} / \mathbf{m l})\end{array}$ & $\begin{array}{c}\text { Absorbance in FRAP } \\
\text { assay }\end{array}$ & $\begin{array}{c}\text { TAC } \\
\left(\mathbf{F e}^{2+} \boldsymbol{\mu m o l} / \mathbf{L}\right)\end{array}$ \\
\hline Vezena & 0,014 & 0,227 & 59 \\
Feferona & 0,019 & 0,228 & 61 \\
Bombona & 0,052 & 0,249 & 90 \\
Control & 0,018 & 0,198 & 18 \\
\hline Sivrija) & & & \\
\hline
\end{tabular}

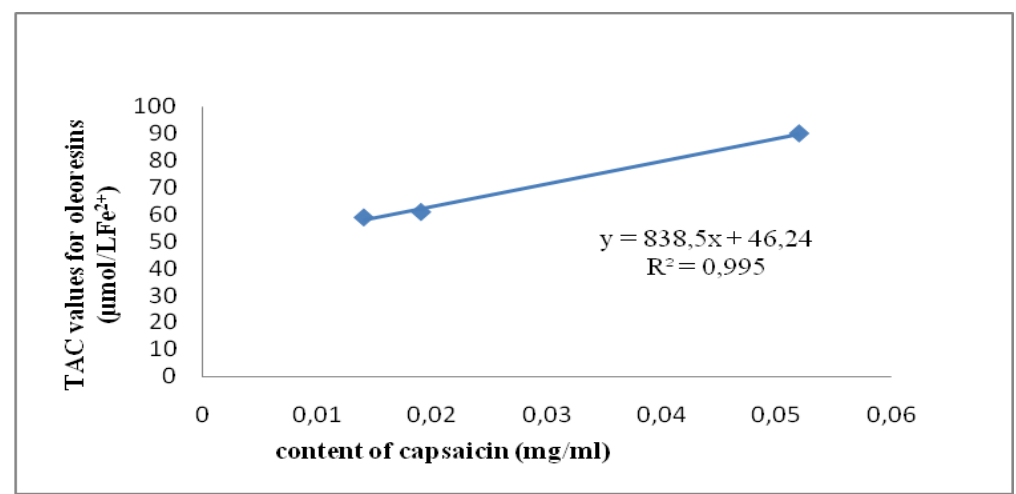

Figure 2 Correlation between capsaicin content and TACs in oleoresins obtained from hot peppers genotypes 
At the end of this study, a comparison between the antioxidant potential of the extracts and the total antioxidant potential of standard solutions of pure capsaicin are shown here in Fig. 4 (Antioxidant potential of capsicum oleoresins vs. capsaicin standard solutions). These measurements were made using water as negative and ascorbic acid as positive control, against ethanol as sample blank.

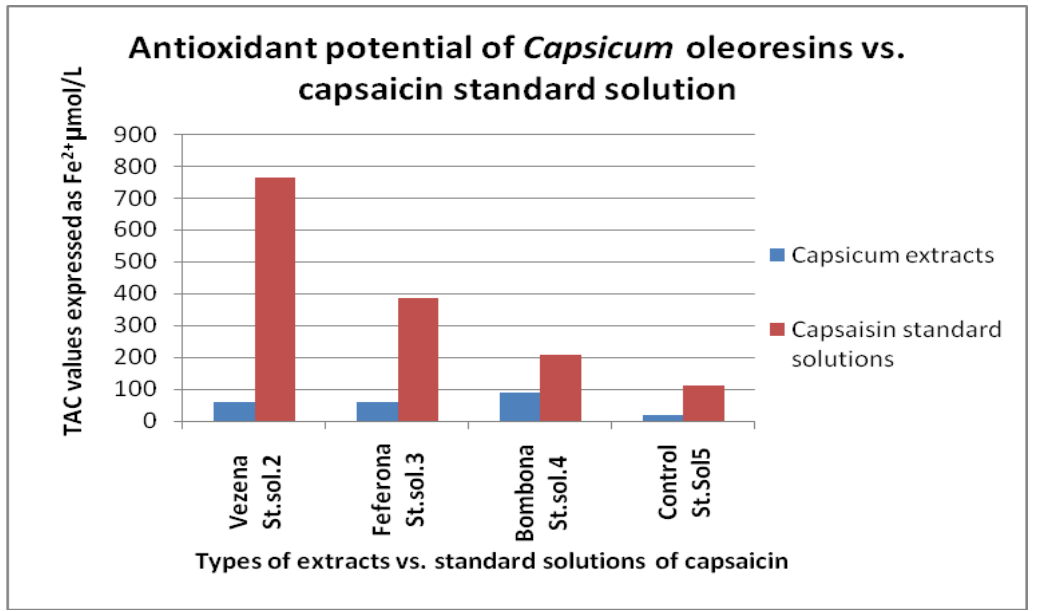

Figure 4 Antioxidant potential of Capsicum oleoresins vs. capsaicin standard solutions

Results are showing good correlation between capsaicin concentration in the extracts and their antioxidant potential. As we can see the genotype Bombona has higher concentration of capsaicin, so we can see, also, higher antioxidant potential, from the examined genotypes of hot peppers. The total antioxidant capacity of the oleoresins obtained from genotypes Vezena and Feferona, (capsaicin concentrations are 0,014 and $0,019 \mathrm{mg} / \mathrm{ml}$ ) are lower than TAC values for pure capsaicin solution with concentration of $0,016 \mathrm{mg} / \mathrm{ml}$. The control, as a mild genotype, has obviously lower antioxidant potential, than hot pepper genotypes. Compared to the TAC values of pure capsaicin the total antioxidant potential of oleoresins is little lower but it has a good correlation with their capsaicin concentration.

\section{CONCLUSION}

As a conclusion: From the previous findings in this study and in the literature, there is a likelihood that pepper fruits may provide the types of nutritional and health benefits associated with the consumption of fresh pepper fruits in general[29-31]. From the results obtained by FRAP (ferric reducing antioxidant power) method, oleoresin obtained from hot peppers genotype that has higher concentration of capsaicin exhibits higher total antioxidant potential than other genotypes, especially from the mild control. This can mean that hot peppers can be used as a potential source of antioxidants because they are rich in capsaicin. This in vitro method for measuring the antioxidant potential is not a specific test for showing the in vivo effects of the antioxidant, because of the known ADME (absorption, distribution, metabolism, elimination) effects on the capsaicin in the human organism. Although the bioactive forms of the capsaicin might not be the one found in the plants, the pepper fruits are still widly used as a source of antioxidants. Because of their often use as a spice and as a food protector further studies into the activity of this compound of peppers are needed to evaluate their potential antioxidative effect and their health benefits for the human organism.

\section{REFERENCESS}

[1]. M. Malgorzata, P. J. Andriena, P., J. Antioxidant Activity of the Main Phenolic Compounds Isolated from Hot Pepper Fruit (Capsicum annuum L.), Agric. Food Chem, 53, 2005, 1750-1756

[2]. M. Peusch, E. Müller-Seitz, M. Petz, A. Müller, E. Anklam, Extraction of capsaicinoids from chillies (Capsicum frutescens L.) and paprika (Capsicum annuum L.) using supercritical fluids and organic solvents, European food research and technology, 1997, 204, 5, 1997, 351-355

[3]. D. Palevitch, L. E. Cracker, Nutritional and medicinal importance of red pepper (Capsicum spp.), J. Herbs Spices Med. Plants, 3, 1995, 55-83

[4]. H.G. Daood, M. Vinkler, F. Markus, E. A. Hebshi, P. A. Biacs, Antioxidant vitamin content of spice red pepper (paprika) asaffected by technological and varietal factors, Food Chem., 55, 1996, 365-372

[5]. N. I. Krinsky, The biological properties of carotenoids, Pure Appl.Chem., 66, 1994, 1003-1010.

[6]. N. I. Krinsky, Carotenoids as antioxidants, Nutrition, 17, 2001 815-817.

[7]. H. Masufuji, H. Nakamura, M. Chino, M. Takeda, Antioxidant activity of capsantin and the fatty acid esters in paprika(Capsicum annuum), J. Agric. Food Chem.,46, 1998, 3468-3472

[8]. R.E Wachtel, Capsaicin, Regist. Anest. Pain Med.,24, 1999, 361-363

[9]. Y.T. Liang, X.Y Tian, J. N. Chen, C. Peng, K. Y. Ma, Y. Zuo, R. 
[10]. Jiao, Y. Lu, Y. Huang, Z. Y. Chen, Capsaicinoids lower plasma cholesterol and improve endothelial function in hamsters, Eur. J. Nutr., 2012, DOI: 10.1007/s00394-012-0344-2.

[11]. H. Manjunatha, K. Srinivasan, Hypolipidemic and antioxidant effects of curcumin and capsaicin in high-fat-fed rats, Can. J. Physiol. Pharmacol., 85(6), 2007, 588-596.

[12]. M. R. Srinivasan, K. Sambiah, M. N. Satyananrayana, M. V. L. Rao, Influence of red pepper and capsaicin on growth, blood constituents and nitrogen balance in rats, Nutr. Res. Int., 21(3), 1980, 457-46.

[13]. S. J. Seo, J. Kim, S. K. Noh, Effect of enteral capsaicin on the lymphatic absorption and fats in rat, Han'guk Sikp'um Yongyang Kwahak Hoechi, 38, 2009, 1712-1717.

[14]. A. R. Josse, S. S. Sherriffs, A. M. Holowerda, R. Andrews, A. W. Staples, S. M. Philips, Effects of capsinoid ingestion on energy expenditure and lipid oxidation at rest and during exercise, Nutr. Metab., 7, 65,2010

[15]. X. J. Luo, J. Peng, Y. J. Li, Recent advances in the study on capsaicinoids and capsinoids, Eur. J. Pharmacol., 650, $2010,1-7$.

[16]. J. PENG, Y. J. Li, The vanilloid receptor TRPV1: role in cardiovascular and gastrointestinal protection, Eur. J. Pharmacol., 627, $2010,1-7$

[17]. S. Rajput, M. Mandal, Antitumor promoting potential of selected phytochemicals derived from spices: a review, Eur. J. Cancer Prev., 21(2), 2012, 205-215.

[18]. I. Díaz-Laviada, N. Rodríguez-Henche, The potential antitumor effects of capsaicin, Prog Drug Res.68, 2014, 181-208

[19]. K. Kogure, S. Goto, M. Nishimura, M. Yasumoto, K. Abe, Ch.Ohiwa, H. Sassa, T. Kusumi, H. Terada, Mechanism of potent antiperoxidative effect of capsaicin, Biochim. Biophys. Acta, 1573, 2002, 84-92

[20]. S.H Kim, J.T. Hwang, H.S Park,D.Y Kwon, M.S Kim, Capsaicin stimulates glucose uptake in C2C12 muscle cells via the reactive oxygen species (ROS)/AMPK/p38 MAPK pathway, Biochem Biophys Res Commun. 439,1, 2013, 66-70

[21]. K. Shetty, Role of proline-linked pentoze phosphate pathway in biosynthesis of plant phenolics for functional food and environmental applications: a review, Process Biochem., 39, 2004, 789-803.

[22]. D. E. Henderson, A. M. Slickman, S. K. Henderson, Quantitative HPLC determination of the antioxidant activity ofcapsaicin on the formation of lipid hydroperoxides of linoleic acid: a comparative study against BHT and melatonin, J. Agric. FoodChem., 47, 1999, 2563-2570.

[23]. M. Contreras-Padilla, E. M. Yahia, Changes in capsaicinoids during development, maturation and sesescence of chile peppers and relation with peroxidase activity, J. Agric. Food Chem., 46, 1998, 2075-2079.

[24]. V. Rafajlovska, R. R. Slavevska, J. Klopcevska, M. Srbinosla, Extraction of Oleoresin from Pungent Red Paprika Under Different Conditions,

[25]. E.C. Wagner, M.T Cahill, A. P. Marshal, Extraction, Purification, and Spectroscopic Characterization of a Mixture of Capsaicinoids, J. Chem. Educ., 88, 2011, 1574-1579

[26]. American College of Toxicology, Final Report on the Safety Assessment of Capsicum Annuum Extract, Capsicum Annuum Fruit Extract, Capsicum Annuum Resin, Capsicum Annuum Fruit Powder, Capsicum Frutescens Fruit, Capsicum Frutescens Fruit Extract, Capsicum Frutescens Resin, and Capsaicin", International Journal of Toxicology, 26, 1, 2007, 3-106

[27]. C. B. Davis,C.E Markey, M.A. Busch, K.W Busch, Determination of capsaicinoids in habanero peppers by chemometric analysis of UV spectral data, J Agric Food Chem. 55, 15, 2007, 5925-33

[28]. I. F. Benzie, J. J. Strain, Ferric reducing/antioxidant power assay: direct measure of total antioxidant activity of biological fluids and modified version for simultaneous measurement of total antioxidant power and ascorbic acid concentration, Methods Enzymol.; 299, 1999, 15-27.

[29]. L. Koleva-Gudeva, V. Rafajlovska, M. Spasenovski, In vivo and in vitro content of capsaicin in pepper Proc. VIII Symposium Biotechnology and Agroindustry, Velika Plana, Serbia, 2004, 252-259

[30]. I. Perucka, M. Materska, Antioxidant activity and contents of capsaicinoids isolated from paprika fruits, Pol. J. Food Nutr.Sci., 12/53, 2, 2003, 15-18.

[31]. AGUIRREZABAL, M. M.; MATEO, J.; DOMINGUEZ, M. C.; ZUMALAC-AREGUI, J. M. (2000), “The effect of paprika, garlic and salt on rancidityin dry sausages", Meat Sci., 54, 77-81.

[32]. T. Aymerich, M. G. Artigas, M. Garriga, J. M. Monfort, M. Hugas, Effect of sausage ingredients and additives on theproduction of enterocin A and B by Enterococcus faecium CTC492. Optimization of in Vitro production and anti-listerial effect in dry fermented sausages, J. Appl. Microbiol., 88, 2000, 686. 